\title{
Der Einzug der Patchwork-Familie in den juristischen Sprachgebrauch - eine Definition
}

Das Erscheinungsbild der deutschen Familie ist in der heutigen Zeit komplex und vielfältig. Die Auflösung und Zerstörung traditioneller Familienstrukturen schreitet stetig voran. Dieser Vorgang ist aber gleichzeitig mit dem Aufbau neuer Strukturen verbunden. So ist das nichteheliche Zusammenleben eines Elternteils mit einem Partner, ${ }^{2}$ der nicht der andere leibliche Elternteil des Kindes ist, eine typische gesellschaftliche Erscheinungsform. Im allgemeinen Sprachgebrauch hat sich für diese Familienform der Begriff Patchwork-Familie etabliert, ${ }^{3}$ wobei es jedoch an einer einheitlichen Definition fehlt. Der Terminus findet sich mittlerweile auch im juristischen Schrifttum wieder. ${ }^{4}$ Es fehlt dabei aber an der rechtlichen Einordnung und Definition der Patchwork-Familie. Auch wurden bisher noch keine, eine solche Familie kennzeichnende, Kriterien herausgearbeitet.

Dieser Problematik stellt sich der Beitrag. Denn das bürgerliche Recht darf sich angesichts der gewandelten Familienverhältnisse nicht vor dieser Familienform verschließen. So stellt sich etwa im Deliktsrecht bei der elterlichen Aufsichtshaftung die Frage, ob und unter welchen Voraussetzungen der neue - nicht leibliche - Elternteil haftet, wenn das Kind seines Partners einen Schaden verursacht.5 Im Familienrecht hat sich der Wandel der tatsächlichen Familienverhältnisse bereits niedergeschlagen. $\int S$ I 682 und I685 BGB, letzterer zudem unlängst reformiert, sind erste Schritte, der veränderten Lebenswirklichkeit vieler Familien Rechnung zu tragen. Dies ist auch die Intention des Beitrages, der die umgangssprachlich als Patchwork-Familie bezeichnete Familienform als eigenständigen Familientyp juristisch definiert.

\section{Das derzeitige Familienbild in Deutschland}

Das deutsche Familienbild hat sich in den letzten Jahrzehnten grundlegend verändert. Dieser Wandel ist gekennzeichnet von anhaltend hohen Scheidungsraten, hohen Wiederverheiratungsquoten und jährlich etwa I 70.000 von Scheidungen ihrer Eltern betroffenen Minderjährigen. ${ }^{6}$ Lebten im früheren Bundesgebiet I 972 noch 93,4\% der Kinder unter I 8 Jahren bei einem zusammenlebenden Ehepaar, so sank diese Zahl im wiedervereinigten Deutschland von 86,9\% I99I, $83,9 \%$ I 996 und 8 I, $4 \% 20007$ auf $79 \%$ im Jahre $2003 .{ }^{8}$ Hingegen stieg in diesem Zeitraum die Zahl der Kinder unter i 8 Jahren, die bei ihrer ledigen, verheiratet

I Besonderer Dank gilt Richterin am AG Ute Flotho, Magdeburg, Staatsanwalt Dr. Ingo Rau, Hannover, und Regierungsrat z. A. Andy Schmidt, Bremen, die diesen Beitrag durch wertvolle Anregungen gefördert haben.

2 Die Begriffe Partner bzw. Elternteil stehen nachfolgend für anders- und gleichgeschlechtliche Lebensgefährten des leiblichen Elternteils des Kindes.

3 Duden, Das große Wörterbuch der deutschen Sprache, 3. A. (I999), Bd. 6, S. 2870; Duden, Die deutsche Rechtschreibung, 23. A. (2004), S. 729; Wahrig, Die deutsche Rechtschreibung, 2005, S. 78 I.

4 Staudinger/Salgo, BGB, I4. A. (2004), \ 1682 Rdnr. 3; ders., FPR 2004, 76; Schindler, DNotZ 2004, 824; Mayer, ZEV 2004, 40 (42); Höfelmann, FamRZ 2004, 745 (75 I); Theurer, MDR 2005, 250 (25 I); Grziwotz, FPR 2005, 283; Bute, FuR 2006, I70 (I74); Standinger/Rauscher, I4. A. (2006) \$ I685 Rn. 9c, 23.

5 Ausführlich dazu Bernau, FamRZ 2006, 82 (85 ff).

6 Salgo, FPR 2004, 76 (78), ohne Quellenangabe.

7 Bundesministerium für Familie, Senioren, Frauen und Jugend (Hrsg.), Die Familie im Spiegel der amtlichen Statistik, 2003, S. 2 I 4 (nachfolgend BMFSFJ).

8 BMFSFJ, unter: www.bmfsfj.de/RedaktionBMFSFJ/Broschuerenstelle/Pdf-Anlagen/Familie-im-Spiegelaktualisierte-Tabellen,property=pdf.pdf in Tabelle $\mathrm{AI}_{\mathrm{I}} \mathrm{I}$, Abfragestand: $30.0 \mathrm{I} .2006$. 
lebten im früheren Bundesgebiet 5,9\% der Kinder in einem solchen Haushalt; nach der Wiedervereinigung waren es in Deutschland dann I99 I I I,5\%, I 996 I 3,6\%, 2000 I 5,7\%9 und 2003 schließlich $17,3 \% .{ }^{10}$ Die klassische Ehe mit Kind ist somit nach wie vor die dominierende Familienform, so wie es der historische Gesetzgeber bei der Normierung der elterlichen Aufsichtshaftung in $\$ 832$ Abs. I BGB als selbstverständlich vorausgesetzt hat. Jedoch befinden sich die Familienformen auf dem Vormarsch, die von diesem ursprünglichen Familienleitbild abweichen. ${ }^{\text {I }}$ Immer mehr Kinder wachsen in Familien auf, in denen nur ein Elternteil das Sorgerecht für sie besitzt. Eine typische, gesellschaftlich akzeptierte Erscheinungsform des heutigen sozialen Lebens ist das Zusammenleben eines Elternteils mit einem Partner, der nicht der andere leibliche Elternteil des Kindes ist. Bei dieser umgangssprachlich als Patchwork-Familie bezeichneten Familienform sind verschiedene Konstellationen denkbar. So können die Mutter oder der Vater oder auch beide Elternteile ein Kind mit in die neue Beziehung bringen. Es kann zudem noch ein gemeinsames Kind aus der neuen Partnerschaft hinzukommen. Angelehnt an den englischen Begriff Patchwork (= Flickwerk) ${ }^{\mathrm{I} 2}$ wird deshalb von einem »Flickenteppich « aus mehreren verschiedenen Familien gesprochen. ${ }^{13}$ Eine negative Wertung wird durch diesen Begriff nicht intendiert.

\section{Die Patchwork-Familie im juristischen Sinn}

BGB und LPartG enthalten weder eine Legaldefinition für die Patchwork-Familie noch bieten sie einen Anhaltspunkt für eine Definition. Erschwerend kommt hinzu, dass die Patchwork-Familie bezüglich des neuen Elternteils, anders als die Ehe oder eine nichteheliche Lebensgemeinschaft mit zwei sorgeberechtigten Eltern ( $($ I626a Abs. I Nr. I BGB), nicht durch Urkunden oder andere Nachweise belegt ist. Die Patchwork-Familie ist daher juristisch durch Abgrenzung zu den bekannten Familienformen zu definieren.

\section{Die Abgrenzung der Patchwork-Familie von der Ehe und der nichtehelichen Lebensgemeinschaft}

Die klassische Familie besteht aus mindestens einem Kind und seinen beiden leiblichen verheirateten, und damit sorgeberechtigten, Eltern, mithin einer ehelichen Lebensgemeinschaft. Sind die beiden leiblichen Eltern des Kindes nicht miteinander verheiratet, so handelt es sich um eine nichteheliche (= eheähnliche)

9 BMFSFJ (Fn. 7), S. 2 I4.

ı० BMFSFJ (Fn. 8), Tabelle A I-I3. Bei diesen Zahlen ist jedoch darauf hinzuweisen, dass bei ledigen Müttern und Vätern auch der andere Elternteil des Kindes zum Haushalt gehören kann, mithin auch die nichteheliche Lebensgemeinschaft der leiblichen Eltern darunter fällt. Auf diese Haushalte entfielen bei Müttern I 972 0,7\%, I 99 I 3,4\%, I 996 4,2\%, 2000 5,2\% und 2003 6,I\%. Bei Vätern betrug diese Zahl I $9720,0 \%$, I 991 1 0,4\%, I 996 0,8\%, 2000 I, I \% und wurde 2003 nicht gesondert erhoben (siehe Fn. 7 f). Eine weitere Differenzierung erfolgt in der amtlichen Statistik nicht.

I I So sieht Bien (in: GEO, Heft 3/2005, S. I 36 [I37]), Mitarbeiter des Deutschen Jugendinstituts in München, das Fundament der traditionellen Familie langsam bröckeln. Zwar sei davon in den meisten ländlichen Regionen noch wenig zu spüren, aber in den Großstädten und vielen Regionen der neuen Bundesländer lasse sich ein Trend zum Zerfall der Kernfamilie ausmachen.

I 2 Der englische Begriff Patchwork (Flickwerk) wird mit aus bunten Flicken zusammengesetzter Stoff bzw. Teppich oder ähnlich übersetzt (Duden, Rechtschreibung [Fn. 2], S. 729; Wahrig, Deutsches Wörterbuch, 7. A. [2000], S. 96I).

I 3 Wikipedia, freie Enzyklopädie, unter: http://de.www.wikipedia.org/wiki/Patchworkfamilie, Abfragestand: 30.01.2006; Gesellschaft für deutsche Sprache, Preisaufgabe Heft 4/2005, unter: www.gfds.de/ preisaufgabe, Abfragestand: 31.10.2005. 
Lebensgemeinschaft, unabhängig davon, ob beide Eltern das Sorgerecht für ihr Kind besitzen. Eine Patchwork-Familie muss infolgedessen wie die Stieffamilie aus einem leiblichen und einem nicht leiblichen Elternteil bestehen, weil das Zusammenleben der leiblichen Eltern mit ihrem gemeinsamen Kind entweder eine eheliche Gemeinschaft (= Ehe) oder eine nichteheliche Lebensgemeinschaft darstellt.

\section{Die Abgrenzung der Patchwork-Familie von der Stieffamilie}

Patchwork-Familie und Stieffamilie sind deshalb voneinander abzugrenzen. ${ }^{14}$ Auch für die Stieffamilie findet sich im BGB und im LPartG keine Legaldefini-

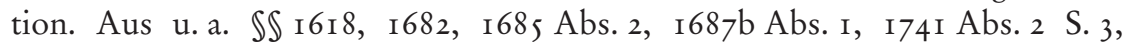
I755 Abs. I BGB, $\mathbb{9}$ Abs. I LPartG ergibt sich jedoch, dass ein Stiefkind das Kind des anderen Ehegatten ist, mithin der Stiefelternteil mit dem leiblichen allein sorgeberechtigten Elternteil des Kindes verheiratet ist. Im juristischen Schrifttum wird der Begriff Patchwork-Familie wiederholt in mehreren Veröffentlichungen genannt. ${ }^{15}$ Grziwotz $^{16}$ und Schindler ${ }^{17}$ verwenden den Begriff wie sich aus dem Kontext ergibt - für Familien, in denen die Partner die Ehe geschlossen bzw. eine Lebenspartnerschaft begründet haben, mithin für Familienkonstellationen, die der Gesetzgeber u. a. in den o. g. Normen berücksichtigt hat. Aus den Ausführungen von Salgo ${ }^{18}$ ergibt sich, dass er vom Terminus neben der Stieffamilie auch eine nichteheliche Lebensgemeinschaft des betreuenden Elternteils mit einer Person verschiedenen, aber auch eine nicht registrierte Lebenspartnerschaft mit einer Person gleichen Geschlechts erfasst sieht. In den juristischen Wörterbüchern fehlt es an einer Erläuterung des Begriffs Patchwork-Familie. ${ }^{19}$ Als Stiefkinder werden dort diejenigen Kinder des anderen Ehegatten definiert, die nicht zugleich eigene Kinder sind. Stiefvater ist der Ehegatte der Mutter, der nicht selbst Vater ist, und Stiefmutter die Ehefrau des Vaters, die selbst nicht Mutter ist. Dabei wird ausdrücklich hervorgehoben, dass die Bezeichnungen Stiefeltern und -kinder keine Rechtsbegriffe sind. ${ }^{20}$

Im allgemeinen Sprachgebrauch wird das Stiefkind als Kind aus einer früheren Ehe des Ehepartners, ${ }^{21}$ als Kind des anderen Ehegatten, ${ }^{22}$ als Kind, das nur von

I4 Die amtliche Statistik des Statistischen Bundesamtes hilft aufgrund der dort vorgenommenen Einteilung der Familienformen bei der Differenzierung zwischen Stieffamilie und Patchwork-Familie nicht weiter. Dort wird bei der Statistik »Ledige Kinder in Familien nach Alter und Familientyp « zwischen drei verschiedenen Familienformen, nämlich Ehepaaren, alleinstehenden Vätern und unverheirateten oder getrenntlebenden Müttern differenziert. Unter die beiden letztgenannten Gruppen fallen Unverheiratete oder verheiratet Getrenntlebende ohne oder mit Lebenspartner im Haushalt, der auch der andere Elternteil des Kindes sein kann (BMFSFJ [Fn. 7], S. 2 I 3 Fn. I). In der Statistik »Familien mit ledigen Kindern nach Familientyp und Alter des jüngsten Kindes « wird zwischen Ehepaaren, Alleinerziehenden und nichtehelichen Lebensgemeinschaften unterschieden. Mit dem letzten Familientyp werden auch Paare erfasst, bei denen beide Partner jeweils eigene Kinder im Haushalt haben; als Alleinerziehende werden Personen ohne Lebenspartner im Haushalt verstanden (BMFSFJ [Fn. 7], S. 2 I 7 Fn. I, 3).

is Höfelmann (FamRZ 2004, 745 [75I]), Theurer (MDR 2005, 250 [25I]) und Bute (FuR 2006, I70 [174]), begnügen sich mit der bloßen Nennung des Terminus.

I6 Grziwotz, FPR 2005, 283.

I7 Schindler, DNotZ 2004, 824 .

I 8 Staudinger/Salgo (Fn. 4), \I682 Rdnr. 3; ders., FPR 2004, 76. Ebenso jüngst Rauscher in: Staudinger, I4. Aufl. (2006), \I685 Rn. 9c, 23.

I9 So in Tilch/Arloth (Hrsg.), Deutsches Rechts-Lexikon, 3. A. (200I), Bd. I und 2; Lexikonredaktion des Verlags F.A. Brockhaus (Hrsg.), Der Brockhaus Recht, 2002; Creifelds, Rechtswörterbuch, I 8. A. (2004); Alpmann/Brockhaus, Fachlexikon Recht, 2004; Köbler, Juristisches Wörterbuch, I3. A. (2005).

20 Creifelds (Fn. I9), S. I 247.

2I Meyers Enzyklopädisches Lexikon, I98 I, Bd. 32, S. 2500 ; Duden, Deutsches Universalwörterbuch, 2. A. (1989), S. I468; Duden, Das große Wörterbuch der deutschen Sprache, 3. A. (I 999), Bd. 8, S. 374I.

22 Meyers Grosses Taschenlexikon, 4. A. (I992), Bd. 2 I, S. I 29. 
einem der Ehegatten abstammt ${ }^{23}$ oder als Sohn oder Tochter des Ehepartners ${ }^{24}$ umschrieben. ${ }^{25}$ Ein Stiefkind ist demnach das Kind eines Elternteils, der durch eine Heirat mit dem leiblichen Vater oder der leiblichen Mutter des Kindes verbunden ist, ohne dass das Kind von jenem Elternteil gezeugt oder geboren worden ist. ${ }^{26}$ Nicht unter den Begriff des Stiefkindes fallen infolgedessen die Kinder, deren leibliches Elternteil mit ihnen in einer nichtehelichen Lebensgemeinschaft lebt. ${ }^{27}$ Die Bezeichnung Patchwork-Familie ist nach Angaben der Gesellschaft für deutsche Sprache e. V. (GfdS) seit den neunziger Jahren im Deutschen geläufig $^{28}$ und wurde als Begriff Mitte der neunziger Jahre geprägt. ${ }^{29}$ Mittlerweile findet man den Begriff Patchwork-Familie auch in den deutschen Wörterbüchern. ${ }^{\circ}$ Sie wird im Duden als Familie definiert, in der außer den gemeinsamen Kindern auch Kinder aus früheren Beziehungen der Mutter bzw. des Vaters oder beider Eltern leben. In der Psychologie wird daher auch von der Fortsetzungsoder Folgefamilie gesprochen. ${ }^{3 \mathrm{I}} \mathrm{Im}$ Wahrig wird die Patchwork-Familie als Familie mit Kindern aus mehreren Verbindungen umschrieben. ${ }^{32}$ Nach Brockhaus 33 und Meyers Grosses Taschenlexikon 34 ist es eine Familie, die durch Scheidung und Wiederverheiratung von Eltern mit Partnern, die ihrerseits Kinder mit in die Ehe bringen, eine neue Art Großfamilie bildet. Die GfdS bezeichnet mit dem Begriff Familien, bei denen die Mutter, der Vater oder beide Eltern ihre jeweiligen Kinder aus vorhergehenden Ehen oder Lebenspartnerschaften in die neue Beziehung einbringen.35 Der Internetenzyklopädie Wikipedia zufolge handelt es sich um Familien, bei denen die Eltern ihre jeweiligen Kinder aus vorhergehenden Ehen oder Lebenspartnerschaften in die neue Beziehung eingebracht haben. Aus der weiteren Erläuterung ergibt sich, dass auch Beziehungen erfasst werden, in denen die neuen Partner die Ehe geschlossen haben. ${ }^{36}$ In einem Internetwörterbuch der Sozialpolitik heißt es unter dem Suchbegriff Patchwork-Familie, dass Familien mit Stiefeltern und -kindern, ggf. Stiefgeschwistern, umgangssprachlich als Patchwork-, Stief- oder Fortsetzungsfamilien bezeichnet werden. ${ }^{37}$ Bringt ein Elternteil ein Kind mit in die neue Partnerschaft, so ist nach Ansicht von Paulsen je nach Weltsicht für diese Familienform der Begriff Stiefoder Patchwork-Familie zu verwenden. ${ }^{38}$ Aus diesen verschiedenen Definitionen wird deutlich, dass umgangssprachlich unter dem Begriff Patchwork-Familie sowohl Beziehungen erfasst werden, bei denen es sich um eine nichteheliche Lebensgemeinschaft handelt, als auch teilweise solche, in denen die neuen Part-

23 Brockhaus, Die Enzyklopädie, 20 A. (1998), Bd. 2 I, S. I44; Meyers Grosses Taschenlexikon, 9. A. (2003), Bd. 22, S. 7200.

24 Wahrig (Fn. I 2), S. I 203.

25 Ausführlich zur juristischen und umgangssprachlichen Definition der Stieffamilie: Carré-Jersch, Das Stiefkindverhältnis im Sorge- und Unterhaltsrecht, Diss. I995, S. I I ff.

26 Bertelsmann Lexikon, I994, Bd. I4, S. 6.

27 Kremer, Das Stiefkind im Unterhaltsrecht, Diss. I998, S. 2 I.

28 Oben Fn. I3. Danach ist ein früher Beleg für den Begriff Patchwork-Familie im Deutschen der Beitrag »Patchworkfamilie und Systemverfall« von Petri in der Tageszeitung taz vom I 2.I 2.I 99I, S. 24.

29 Der Brockhaus multimedial (2004) unter dem Stichwort: Patchwork-Familien.

30 Noch keine Erwähnung findet die Patchwork-Familie in: Langenscheidt, Großwörterbuch Deutsch als Fremdsprache, Hrsg. Götz/Haensch/Wellmann, 2003; Paul, Deutsches Wörterbuch, Io. A. (2002).

3I Duden, Wörterbuch (Fn. 3), S. 2870. Im Duden, Rechtschreibung (Fn. 3), S. 729, heißt es: Familie, in der außer den gemeinsamen Kindern auch Kinder aus früheren Beziehungen der Eltern leben.

32 Wahrig (Fn. 3), S. $78 \mathrm{I}$.

33 Oben Fn. 29.

34 Meyers Grosses Taschenlexikon, 9. A. (2003), Bd. 17, S. 5582.

35 Oben Fn. I3.

36 Oben Fn. I3.

37 Wörterbuch der Sozialpolitik, unter: www.socialinfo.ch/cgi-bin/dicopossode/show/cfm?id=448, Abfragestand: 3I.10.2005.

38 Paulsen, GEO, Heft $3 / 2005$, S. I36. 
ner die Ehe miteinander eingegangen sind. Aufgrund der Gesetzessystematik von BGB und LPartG sowie dem juristischen Sprachgebrauch ist die rechtswissenschaftliche Abgrenzung zwischen Patchwork-Familie und Stieffamilie jedoch am Merkmal der Eheschließung der neuen Partner vorzunehmen.

\section{Die Abgrenzung der Patchwork-Familie von der bloßen Haus- und Wohngemeinschaft}

Hinsichtlich der Beziehungsintensität ist die Patchwork-Familie von einer bloßen Haus- und Wohngemeinschaft zu unterscheiden und abzugrenzen. Das Band zwischen den rechtlich nicht miteinander verbundenen neuen Partnern muss stärker als bei einer solchen Zweckgemeinschaft geknüpft sein, um es mit Rechten und Pflichten gegenüber Dritten belasten zu können. Dabei hilft ein Blick in das Mietrecht, welches sich ebenfalls den Veränderungen und Weiterungen der Formen menschlichen Zusammenlebens stellen muss. Dort erfolgt die Differenzierung beim Eintrittsrecht des überlebenden Partners einer eheähnlichen (= nichtehelichen) Gemeinschaft in das bestehende Mietverhältnis anhand der Tatbestandsvoraussetzung "auf Dauer angelegter gemeinsamer Haushalt«, \563 Abs. 2 S. 4 BGB. Der BGH hat mit Beschluss v. I3.0I.1993 in einem Rechtsentscheid zur analogen Anwendung des $\$ 569$ a Abs. 2 S. I BGB a. F., der Vorgängerregelung des $\ 563 \mathrm{BGB}$, objektive und nachprüfbare Kriterien aufgestellt, die eine sachgerechte und nachprüfbare Abgrenzung einer solchen Lebensgemeinschaft zu anderen, weniger intensiven Formen des Zusammenlebens ermöglichen. Danach muss die Lebensgemeinschaft auf Dauer angelegt sein, keine weiteren Bindungen gleicher Art zulassen und sich durch innere Bindungen auszeichnen, die ein gegenseitiges Einstehen der Partner füreinander begründen, also über eine reine Haushalts- und Wirtschaftsgemeinschaft hinausgehen. Die für eine solche Feststellung heranzuziehenden Indizien lassen sich nicht erschöpfend aufzählen. Von Bedeutung sind aber vor allem die Dauer des Zusammenlebens, die Versorgung von Kindern im gemeinsamen Haushalt und die Befugnis, über Einkommen und Vermögensgegenstände des Partners zu verfügen. Eine vorübergehende Partnerschaft scheidet demnach als Lebensgemeinschaft aus, da es ihr am Merkmal der Dauerhaftigkeit mangelt.39 In den Gesetzesmaterialien zu $\int 563$ BGB wird ausdrücklich hervorgehoben, dass das Eintrittsrecht allen Lebenspartnern ungeachtet ihrer sexuellen Orientierung zusteht und es auf das Vorliegen geschlechtlicher Beziehungen nicht ankommt. Im Übrigen lehnt sich die Gesetzesbegründung bei der Erläuterung des Merkmals auf Dauer angelegter gemeinsamer Haushalt an die vorgenannten Ausführungen des $B G H$ an. $4^{\circ}$ Aufgrund des Wortlauts von $\ 563$ Abs. 2 S. 4 BGB soll es nicht zwingend darauf ankommen, dass der gemeinsame Haushalt bereits seit längerer Zeit besteht. ${ }^{\mathrm{I}}$ Leben die Partner bereits längere Zeit zusammen, kann infolgedessen die Dauerhaftigkeit der Beziehung unproblematisch unterstellt werden. Anderenfalls ist sie nach den Umständen des Einzelfalls zu beurteilen. Gewichtige Indizien sind dabei, dass die Partner nur einen einzigen gemeinsa-

$39 B G H Z$ I 2 I, I I6 (I 24 f.) = NJW I993, 999 (I00I) v. I 3.0I.I993. Der Senat schloss dabei gleichgeschlechtliche Partnerschaften ausdrücklich aus.

40 Mietrechtsreformgesetz v. 29.3.200I, BT-Drucks. I4/4553, S. 6 I.

4I Schmidt-Futterer/Gather, Mietrecht, 8. A. (2003), \563 Rdnr. 29; Emmerich/Sonnenschein/Rolfs, Mietrecht, 8. A. (2003), $\ 563$ Rdnr. I 2; Blank/Börstinghaus, Miete, 2. A. (2004), $\ 563$ Rdnr. 47; MünchKomm/Häublein, 4. A. (2004), $\$ 563$ Rdnr. I4; einschränkend Hinz, ZMR 2002, 640 (642), der auf unterschiedliche Interpretationsmöglichkeiten, nämlich zum einen auf die bestehende Dauer der bisherigen Haushaltsführung und zum anderen auf die gegenwärtigen Absichten der Beteiligten, verweist. 
men Hausstand führen, also keiner der beiden Partner mehr über eine weitere Wohnung verfügt, und eine wechselseitige Versorgung besteht. ${ }^{2}$ Für einen auf Dauer angelegten Haushalt spricht auch eine gemeinsame Freizeit- und Urlaubsplanung43 oder Hausratversicherung.

Diese Kriterien des Mietrechts können zur Abgrenzung der Patchwork-Familie von einer bloßen Wohngemeinschaft herangezogen werden, da sich Erstere von der nichtehelichen Lebensgemeinschaft nur durch die fehlende leibliche Elternschaft eines Partners unterscheidet. Die Abgrenzung erfolgt dabei anhand aller Umstände des Einzelfalls. Ziehen ein Mann oder eine Frau mit ihrem neuen Partner, der ein Kind mit in die Beziehung bringt, in eine gemeinsame Wohnung mit gemeinschaftlicher Haushaltsführung zusammen, handelt es um eine Lebensgemeinschaft. Das gilt ab dem ersten Tag des Zusammenlebens. Der hinzukommende Partner wird realistischerweise in das bestehende Familienleben des leiblichen Elternteils mit seinem Kind integriert. Auch entspricht es der Lebenserfahrung und -wirklichkeit, dass der neue Partner neben dem leiblichen Elternteil elterliche Aufgaben, Funktionen und Rollen übernimmt. Dasselbe gilt natürlich auch, wenn jeder der beiden Partner ein Kind mit in die Beziehung bringt.

\section{Die Definition der Patchwork-Familie}

Die Patchwork-Familie im juristischen Sinn ist eine im gemeinsamen Haushalt lebende, nichteheliche Lebensgemeinschaft mit mindestens einem Kind, welches nur vom dafür sorgeberechtigten Partner abstammt. Die kleinstmögliche Patchwork-Familie setzt somit drei Personen voraus. Der nicht leibliche, sorgerechtslose Elternteil ist in Bezug auf das Kind des Partners als Patchwork-Elternteil zu bezeichnen. Gehen die neuen Partner miteinander die Ehe ein, so ist von einer Stieffamilie zu sprechen, vom nicht leiblichen Elternteil mithin als Stiefelternteil. Die juristische Definition der Patchwork-Familie ist somit enger als die umgangssprachliche Definition, da die Letztere auch die Stieffamilie unter den Begriff Patchwork-Familie fasst. Das Abgrenzungsmerkmal der PatchworkFamilie zur nichtehelichen Lebensgemeinschaft besteht darin, dass bei ihr mindestens ein Kind nur von einem der beiden Partner abstammt, während in einer nichtehelichen Lebensgemeinschaft nur gemeinsame Kinder beider Partner leben.

Die Drei-Generationenfamilie, auch als Großfamilie bezeichnet, wird von der juristischen Definition der Patchwork-Familie nicht erfasst. Es fällt nur die Zwei-Generationenfamilie unter den juristischen Terminus. Denn die - hier zur Abgrenzung herangezogenen - Begriffe Ehe, Stieffamilie und nichteheliche Lebensgemeinschaft umfassen im bürgerlichen Recht ebenfalls nur die ZweiGenerationenfamilie. Zudem wird auch im allgemeinen Sprachgebrauch die Bezeichnung Patchwork-Familie nur für die aus Eltern und Kind bestehende Kleinfamilie verwandt. $44 \mathrm{Ob}$ die weitere Entwicklung der Familienstrukturen eine Erweiterung oder Differenzierung der juristischen Definition der Patchwork-Familie auf die Drei-Generationenfamilie notwendig machen wird, bleibt abzuwarten. Derzeit ist eine solche Begriffsbildung jedenfalls nicht erforderlich. 


\section{Der Familienbegriff in Art. 6 Abs. I GG}

Die Veränderung der gesellschaftlichen Familienstruktur hat auch vor dem verfassungsrechtlichen Familienschutz nicht Halt gemacht. Die Interpretation des Familienbegriffs in Art. 6 Abs. I GG, die bei der durch die Ehe begründeten Familie begann, ist stetig erweitert worden.

a) Der Familienbegriff des Bundesverfassungsgerichts

Im Stichentscheid-Urteil v. 29.07.1959 bezeichnete das Bundesverfassungsgericht die Familie als umfassende Gemeinschaft von Eltern und Kindern, in der den Eltern vor allem das Recht und die Pflicht zur Pflege und Erziehung der Kinder erwachsen. Es verwies darauf, dass der Ordnungskern dieses Instituts für das allgemeine Rechtsgefühl und -bewusstsein unantastbar sei.45 Noch deutlicher formulierte das Bundesverfassungsgericht den Zusammenhang der Institute Ehe und Familie im Familiennachzug-Beschluss v. I 2.05. I987. Danach sei die Ehe die rechtliche Form umfassender Bindung zwischen Mann und Frau. Sie bilde die alleinige Grundlage einer vollständigen Familiengemeinschaft und als solche die Voraussetzung für eine bestmögliche körperliche, geistige und seelische Entwicklung von Kindern. ${ }^{6}$ Trotz dieser Formulierungen, die noch heute Bestand haben, sieht das Bundesverfassungsgericht seit einem Beschluss v. 23.10.195847 die aus Mutter und nichtehelichem Kind bestehende Familiengemeinschaft vom Schutzbereich des Art. 6 Abs. I GG erfasst und hat diesen allgemein anerkannten Schutz mehrfach bestätigt. ${ }^{8}$ Seit dem Wegfall des $\int$ I 589 Abs. 2 BGB,49 der eine Verwandtschaft zwischen dem nichtehelichen Kind und seinem Vater ausschloss, sieht das Bundesverfassungsgericht auch diese Beziehung vom Familienbegriff in Art. 6 Abs. I GG erfasst. ${ }^{\circ} \mathrm{Um}$ den nichtehelichen Vater und sein leibliches Kind als eine von Art. 6 Abs. I GG geschützte Gemeinschaft anzusehen, ist aber ein Zusammenleben der beiden erforderlich. Das stellte das Gericht I98 I in einem Beschluss ausdrücklich fest. ${ }^{5 \mathrm{I}}$ Zuvor ergab sich dies für das Bundesverfassungsgericht aus dem Schutzgedanken des Art. 6 Abs. I GG, wonach dieser das Zusammenleben von Eltern und Kindern in einer häuslichen Gemeinschaft schützt. ${ }^{2}$ Fehlt es an einem solchen Zusammenleben, kann sich der nichteheliche Vater nicht auf den Schutz der Familiengemeinschaft nach Art. 6 Abs. I GG berufen. 53 , 54 In seiner Entscheidung zum Umgangsrecht des biologischen Vaters vom 09.04.2003 führt das Bundesverfassungsgericht nunmehr aus, dass auch der leibliche, aber nicht rechtliche Vater eines Kindes mit diesem eine unter dem Schutz des Art. 6 Abs. I GG stehende Familie bildet, wenn zwischen

\footnotetext{
45 BVerfGE Io, 59 (66) v. 29.07.1959.

$46 B \operatorname{VerfGE} 76$, I (5I) v. I 2.05.1987.

47 BVerfGE 8, 210(215) v. 23.10.1958.

48 BVerfGE I8, 97 (Ios f) v. 30.06.1964; 25, I67 (I96) v. 29.01.1969; 45, I04 (I 23) v. 08.06.1977.

$49 \$$ I589 Abs. 2 BGB, aufgehoben durch das NEhelG v. I9.08.1969 (BGBl. I I969, S. I 243) zum 01.07.1970, lautete i.d. F. v. ог.01.1900: »Ein uneheliches Kind und dessen Vater gelten nicht als verwandt.

50 $B \operatorname{VerfGE} 45$, 104 (123) v. 08.06.1977.

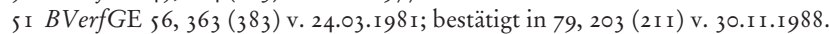

52 BVerfGE 28, I04 (I I 2) v. I 8.03.1970; 48, 327 (339) v. 3 I.05.1 978.

53 BVerfGE 79, 203 (2 I I) v. 30.I I.I988.

54 An das Merkmal der häuslichen Gemeinschaft knüpft auch der Schutz von Ehe und Familie in den jungen LVerf der drei mitteldeutschen Bundesländer an. So lautet Art. 24 Abs. 2 S. I LVerf LSA »Wer in häuslicher Gemeinschaft für Kinder oder Hilfsbedürftige sorgt, verdient Förderung und Entlastung.« Ähnlich sind die Formulierungen in Art. 22 Abs. 2 SächsVerf und Art. 17 Abs. 2 LVerf Thür.
} 

aus, dass der leibliche Vater zumindest eine Zeit lang tatsächlich Verantwortung für sein Kind getragen hat und daraus eine soziale Beziehung zwischen ihm und dem Kind entstanden ist.55 Die von ihm neu eingeführte Begriffskategorie »sozial-familiäre Beziehung « leitet das Bundesverfassungsgericht somit aus der Wahrnehmung tatsächlicher Verantwortung ab, ohne diese näher zu konkretisieren. Eine solche Übernahme wird nach Auffassung des Gesetzgebers i. d. R. anzunehmen sein, wenn der Vater mit dem Kind längere Zeit in häuslicher Gemeinschaft gelebt hat. ${ }^{6} \mathrm{Zu}$ den in einer Familiengemeinschaft aufwachsenden und damit vom Familienbegriff des Bundesverfassungsgericht erfassten Kindern gehören auch Stief-, Adoptiv- und Pflegekinder.57 Art. 6 Abs. I GG erfasst hingegen nach Ansicht des Bundesverfassungsgerichts nicht den Schutz der Generationen-Großfamilie. Familie i.S. v. Art. 6 Abs. I GG bedeutet danach grundsätzlich die in der Hausgemeinschaft geeinte engere Familie, das sind die Eltern mit ihren Kindern, also die moderne Kleinfamilie..$^{8}$

Der verfassungsrechtliche Familienschutz erfordert somit das Vorhandensein zumindest eines Kindes. Familie i. S. d. Art. 6 Abs. I GG ist nach dem Bundesverfassungsgericht die häusliche Gemeinschaft zwischen Eltern bzw. Elternteil und Kind, die auf einer Ehe beruhen kann, aber nicht auf ihr beruhen muss. Jede häusliche Lebensgemeinschaft eines leiblichen oder rechtlichen Elternteils mit seinem Kind fällt somit in den Schutzbereich des Familienbegriffs in Art. 6 Abs. I GG.59

\section{b) Der Familienbegriff im Schrifttum}

Das Schrifttum referiert die vorgenannte Rechtsprechung des Bundesverfassungsgerichts zum Familienbegriff des Art. 6 Abs. I GG überwiegend zustimmend und weist dabei auf die Bedeutung der Familie für den personellen Fortbestand der Gesellschaft hin. Obwohl der Zweck der Volkserhaltung kein eigenständiger Legitimationsgrund für die verfassungsrechtliche Wertentscheidung in Art. 6 GG sei, werde durch die Regeneration der Gesellschaft zugleich ein Beitrag zur Bevölkerungspolitik geleistet. ${ }^{60}$ Ein restriktiver Familienbegriff, ${ }^{61}$ wonach nur verheiratete Eltern mit Kindern dem verfassungsrechtlichen Familienleitbild entsprechen, wird von der ganz herrschenden Lehre aufgrund

58 BVerfGE I0, 59 (66) v. 29.07.1959; 48, 327 (339) v. 31.05.1978; für das Verhältnis Großeltern-Enkelkind offengelassen in BVerfGE 39, $316(326)$ v. 06.05.1975.

$59 B \operatorname{VerfGE~80,~8I~(90~f)~v.~I~8.04.1989;~bestätigt~in~99,~I~} 45$ ( I 56) v. 29. I0.1 998. Den Schutzumfang des Art. 6 Abs. I GG stuft das Bundesverfassungsgericht je nach dem Entwicklungsstand der Familie als Lebensund Erziehungs-, als Haus- oder als Begegnungsgemeinschaft ab. Danach schützt Art. 6 Abs. I i. V. m. Abs. 2 GG die Familie zunächst und zuvörderst als Lebens- und Erziehungsgemeinschaft, da die leibliche und seelische Entwicklung der Kinder in der Familie und der elterlichen Erziehung eine wesentliche Grundlage findet. Mit wachsender Handlungs- und Entscheidungsfähigkeit des Kindes treten Verantwortlichkeit und Sorgerecht der Eltern zurück, so dass die Lebensgemeinschaft dadurch zur bloßen Hausgemeinschaft werden kann. Mit der Auflösung der Hausgemeinschaft wandelt sich die Familie sodann zur bloßen Begegnungsgemeinschaft.

60 BerlinKomm/Burgi, GG, Loseblatt Grundwerk (2000), Art. 6 Rdnr. 38; BK/Pirson, GG, Loseblatt Io I. Lfg. (2002), Art. 6 Abs. I Rdnr. 29 f; Steiger, VVDStRL 45, 55 (85); Zuleeg, NVwZ I986, 800 (806 f).

6I Lecheler (in HStR VI, I989, $\int$ I 33 Rdnr. 42-47; DVBl I986, 905 [907]); v. Campenhausen (in VVDStRL $45,7[2 \mathrm{I} f f])$. 
des Schutzzweckes von Art. 6 Abs. I GG abgelehnt. ${ }^{62}$ Mitunter wird der Familienbegriff des Art. 6 Abs. I GG unabhängig vom Zusammenleben auf den Vater und sein nichteheliches Kind erstreckt. ${ }^{6}$ Überwiegend wird jedoch mit Verweis auf die Rechtsprechung des Bundesverfassungsgerichts für ausschlaggebend erachtet, ob die uneheliche Vaterschaft zu tatsächlichen Beziehungen zum Kind geführt hat, die für familiäre Beziehungen charakteristisch sind, wie ein Zusammenleben von Vater und Kind, ${ }^{64}$ oder aber, dass der Vater zumindest tatsächlich Verantwortung für sein Kind trägt. ${ }^{65}$

Entgegen dem Bundesverfassungsgericht will das Schrifttum den Schutz des Art. 6 Abs. I GG nicht auf die Kleinfamilie beschränken. Die Erstreckung des Familienbegriffs in Art. 6 Abs. I GG auf die Generationen-Großfamilie wird mit der konsequenten Anwendung des Kriteriums einer gewachsenen Bindung ${ }^{66}$ sowie der blutsmäßigen Verwandtschaft, sozialer Tradition und einem umfassenderen Verständnis von Familie im bürgerlichen Recht ${ }^{67}$ begründet. ${ }^{68}$ Jedoch setzt diese Erweiterung des Familienschutzes damit ebenfalls voraus, dass mindestens ein Kind vorhanden ist. Der zumeist geringeren, in ihrer Intensität abnehmenden Nähe der Familienmitglieder zueinander im Rahmen der Großfamilie könne und müsse bei der Schutzintensität und der Konkretisierung der Schutzinhalte des Art. 6 Abs. I GG Rechnung getragen werden. ${ }^{69}$

\section{Die Beachtung der veränderten Familienstruktur im bürgerlichen Recht}

Die Umwälzungen der Familienverhältnisse haben auch im Familienrecht des BGB ihren Niederschlag gefunden. ${ }^{70}$ Der Gesetzgeber beachtet zunehmend die Veränderung der Familienstrukturen, was sich aus der Schaffung der Regelungen in $\iiint_{1682}$, I685 BGB ergibt. Das Familienrecht ist in diesem Bereich noch nicht wieder zur Ruhe gekommen, was durch die erneute Änderung des \I685 Abs. 2 BGB zum 30.04.2004 deutlich wird.

62 v. Mangoldt/Klein/Robbers, Das Bonner Grundgesetz, Bd. I, 5. A. (2005), Art. 6 Abs. I Rdnr. 90; BK/ Pirson (Fn. 60), Art. 6 Abs. I Rdnr. 24; Maunz/Dürig/Badura, GG, Loseblatt Lfg. 44 (Februar 2005), Art. 6 Abs. I Rdnr. 6r; HVerfR/E. M. v. Münch, 2. A. (I994), \$9 Rdnr. I3; MünchKomm/Koch, Bd. 7 (Familienrecht I), 4. A. (2000), Einl FamR Rdnr. I9I. Stellvertretend sei auf die Argumentation von Robbers (aaO.) verwiesen. Danach erfülle die Gemeinschaft des überlebenden Ehegatten mit den Kindern grundsätzlich in besonderem Maße die soziale, schutzwürdige Funktion der Familie in der Stabilisierung bei seelischen Krisensituationen. Den Beteiligten den rechtlichen Schutz zu entziehen, wenn sie ihn am dringendsten brauchen, könne kaum sinnvolles verfassungsrechtliches Ziel sein. Dasselbe gelte für das Verhältnis zwischen nichtehelicher Mutter bzw. Vater und dem nichtehelichen Kind.

63 v. Mangoldt/Klein/Robbers (Fn. 62), Art. 6 Abs. I Rdnr. 90; Pieroth/Schlink, Grundrechte - Staatsrecht II, 20. A. (2004), Rdnr. 642; v. Münch/Kunig/Coester-Waltjen, GG, 5. A. (2000), Art. 6 Rdnr. I I.

64 BK/Pirson (Fn. 60), Art. 6 Abs. I Rdnr. 24; Dreier/Gröschner, GG, Bd. I, 2. A. (2004), Art. 6 Rdnr. 77.

65 Maunz/Dürig/Badura (Fn. 62), Art. 6 Abs. I Rdnr. 6r.

66 Dreier/Gröschner (Fn. 64) Art. 6 Rdnr. 77.

67 v. Mangoldt/Klein/Robbers (Fn. 62), Art. 6 Abs. I Rdnr. 86-88.

68 Auf den Einzelfall abstellend: BK/Pirson (Fn. 60), Art. 6 Abs. I Rdnr. 21; ohne nähere Begründung: Maunz/Dürig/Badura (Fn. 6I), Art. 6 Abs. I Rdnr. 6o; v. Münch/Kunig/Coester-Waltjen (Fn. 63), Art. 6 Rdnr. I I; Jarass/Pieroth, GG, 7. A. (2004), Art. 6 Rdnr. 4; Pieroth/Schlink (Fn. 63), Rdnr. 643; Lecheler, DVBl i 986,905 (908).

69 v. Mangoldt/Klein/Robbers (Fn. 62), Art. 6 Abs. I Rdnr. 89; v. Münch/Kunig/Coester-Waltjen (Fn. 63), Art. 6 Rdnr. 35 .

70 Nach Ansicht von Salgo (in Staudinger (Fn. 4), \I682 Rdnr. 3; ders., FPR 2004, 76) konnte sich der Gesetzgeber noch nicht zu einer umfassenden Berücksichtigung von Stief- und Pflegekindschaft, mithin einer generellen Berücksichtigung von sozialer Elternschaft im Recht durchringen. Dies verwundere aber angesichts der Vielfalt und der Komplexität des Zusammenlebens in neu zusammengesetzten Familienformen nicht. Die Regulierungs- und Steuerungsfähigkeit des Rechts stoße in diesem Dschungel von Beziehungen schnell an Grenzen, weshalb die Rechtspolitik in diesem Bereich durch zwangsläufig punktuelles Stückwerk mit wichtigen Einzelregelungen gekennzeichnet sei. 
Durch den mit dem SorgeRG ${ }^{11}$ eingeführten und durch das KindRG ${ }^{72}$ modifizierten $\ 1_{632}$ Abs. 4 BGB wurde für die Pflegefamilie ein gewisser Bestandsschutz geschaffen. Dieser Schutzgedanke schlägt sich auch in dem durch das KindRG eingeführten und durch das LPartG73 erweiterten $\$ I682 BGB - der sich an $\ \mathrm{I}_{632}$ Abs. 4 BGB orientiert - nieder, der das Zusammenleben in einer Familie in seinem tatsächlichen Bestand schützt. Mit der Vorschrift hat der Gesetzgeber der Erkenntnis Rechnung getragen, dass oft der weitere Verbleib des Kindes nach dem Weg- bzw. Ausfall des sorgeberechtigten Elternteils (in den Fällen der $\$ \int$ I 678, I680 und I68 I BGB) in der alten Familie unter Kontinuitätsgesichtspunkten bessere Entwicklungsmöglichkeiten bietet als der Übergang zu dem ferner stehenden Elternteil.74 Der Kreis der von \I682 BGB erfassten Personen, die mit dem bisher sorgeberechtigten Elternteil eine Hausgemeinschaft gebildet haben, ist begrenzt. Er umfasst den Stiefelternteil, den früheren Lebenspartner und die nach $\int$ I $_{6} 5$ s Abs. I BGB umgangsberechtigten Großeltern bzw. volljährigen Geschwister, die bisher Sorgebefugnisse mit dem anderen Elternteil ausgeübt und Verantwortung getragen haben. Bewusst ausgeklammert aus dem geschützten Personenkreis blieb der nichteheliche Lebenspartner des betreuenden Elternteils, da nach Ansicht des Gesetzgebers ein eindeutiger und klarer Anknüpfungspunkt für die gesetzliche Umschreibung dieses Personenkreises fehlt. ${ }^{75}$

Ebenfalls durch das KindRG wurde $\$$ I685 BGB als Teil der reformierten Gesamtregelung des Umgangsrechts neu in das BGB eingefügt. Diese im Gesetz gänzlich neue Regelung basiert auf dem Gedanken, die über die Kleinfamilie hinausgehenden Sozialbeziehungen des Kindes rechtlich zu stärken. Während $\int$ I685 in Abs. I BGB für das Umgangsrecht für Großeltern und Geschwister ${ }^{6}$ allein an das Verwandtschaftsverhältnis anknüpfte, erforderte Abs. 2 eine gewachsene soziale Familienbeziehung in Form einer länger andauernden häuslichen Gemeinschaft für das Umgangsrecht des Stiefelternteils und der Pflegeeltern. Durch $\mathbb{S}$ I68 5 Abs. 2 BGB sollte die gänzliche Beseitigung der gewachsenen sozialen Familienbeziehung, in der das Kind gelebt hat, vermieden werden.77 Anderen als den in $\$ I 685 BGB ausdrücklich genannten Personen, insbesondere dem nichtehelichen Partner des Elternteils, stand nach dem Willen des Gesetzgebers unter keinen Umständen ein Umgangsrecht zu. Dies galt selbst dann, wenn zu einer solchen Person aufgrund einer längeren häuslichen Gemeinschaft eine soziale Familienbeziehung bestand. $7^{8}$

Mit seinem Urteil v. 09.04.2003 erklärte das Bundesverfassungsgericht \I685 Abs. 2 BGB insoweit mit Art. 6 Abs. I GG für unvereinbar, als er den leiblichen Vater eines Kindes, der eine sozial-familiäre Beziehung zu diesem habe, auch dann von dem Kindesumgang ausschließt, wenn der Umgang dem Kindeswohl

\footnotetext{
7i SorgeRG v. i 8.07.1979, BGBl. I i979, S. го6г.

72 KindRG v. I6.1 2.1997, BGBl. I I997, S. 2942.

73 LPartG v. I6.02.200I, BGBl. I 200I, S. 266.

74 MünchKomm/Finger, BGB, 4. A. (2002), \I682 Rdnr. I f.

75 BT-Drucks. I3/4899, S. 66; zustimmend, wenn auch kritsch: Staudinger/Salgo (Fn. 4), \I682 Rdnr. I, 3 a. E.; ders., FPR 2004, 76 f; a. A. MünchKomm/Finger (Fn. 74), \I682 Rdnr. Io, der den nichtehelichen Lebenspartner durch eine analoge Anwendung der $\$ \int 1682$, I685 Abs. 2 BGB mit in den Personenkreis einbeziehen will; ebenso Palandt/Diederichsen, BGB, 65 A. (2006), \I682 Rdnr. 2 mit Verweis auf $\$$ I685 Rdnr. 6.

76 Nach Ansicht von Theurer (MDR 2005, 250 [25I ff m. w. N.]) räumt die Rechtsprechung den Großeltern und anderen dritten Personen dennoch ein Umgangsrecht mit Kindern nur sehr zögerlich ein.

77 Staudinger/Rauscher, BGB, I3. A. (2000), $\mathbb{1}$ I685 Rdnr. I f, 8, I0.

78 BT-Drucks. I3/4899, S. 69, I06 f; OLG Bamberg, FamRZ I999, 8 Io v. 29.01.1999; a. A. und für eine Einbeziehung des nichtehelichen Lebensgefährten: Staudinger/Rauscher (Fn. 77), \I685 Rdnr. I7; Palandt/ Diederichsen, BGB, 63 A. (2004), \I685 Rdnr. 7; MünchKomm/Finger (Fn. 74), $\$ I685 Rdnr. I I f.
} 
diene. Die Verfassungshüter gaben dem Gesetzgeber auf, bis zum 30.04.2004 eine verfassungsgemäße Neuregelung zu treffen.79 Dem kam der Gesetzgeber fristgemäß nach ${ }^{80}$ und ging sogar noch über die Vorgaben des Bundesverfassungsgerichts hinaus. Bei der Neufassung der $\$ \iint_{1600}$ Abs. 3, I685 Abs. 2 BGB wurde die vom Bundesverfassungsgericht angenommene Wechselwirkung zwischen sozial-familiärer Beziehung und Verantwortungsübernahme aufgegriffen. Der Rechtspraxis gab er durch die Aufnahme von Regelannahmen für den Begriff der sozial-familiären Beziehung im BGB Orientierungshilfen. ${ }^{8}$ Eine Übernahme tatsächlicher Verantwortung - und damit eine sozial-familiäre Beziehung - liegt danach i. d. R. vor, wenn der rechtliche Vater mit der Mutter des Kindes verheiratet ist ( $\int 1600$ Abs. 3 S. 2 BGB) oder er bzw. die enge Bezugsperson mit dem Kind längere Zeit in häuslicher Gemeinschaft zusammengelebt hat ( $\int 1600$ Abs. 3 S. 2 BGB bzw. $\int 1685$ Abs. 2 S. 2 BGB). Der Gesetzgeber hat dabei offen gelassen, welche Zeitdauer dafür erforderlich ist. Luthin nennt 6 Monate als möglichen Richtwert. ${ }^{82}$ Schwab, der in $\ 1685$ Abs. 2 BGB einen verfassungswidrigen Eingriff in das Elternrecht erblickt, gibt zu bedenken, dass sich Kind und sorgeberechtigtes Elternteil einer Ansammlung von Umgangsberechtigten gegenüber sehen können, wenn der Sorgeberechtigte mit mehreren Personen hintereinander eine gewisse Zeit zusammengelebt hat. ${ }^{83}$ Nunmehr gehört sowohl der leibliche, aber nicht rechtliche Vater des Kindes als auch der ehemalige nichteheliche Lebensgefährte des Elternteils zum Kreis der Umgangsberechtigten in $\$$ I 685 Abs. 2 BGB, wenn er die übrigen Voraussetzungen erfüllt.

\section{Wo steht die Patchwork-Familie?}

Die Patchwork-Familie fällt in ihrer Gesamtheit nicht unter den verfassungsrechtlichen Familienbegriff in Art. 6 Abs. I GG. Geschützt durch Art. 6 Abs. I GG wird nur die familiäre Beziehung des leiblichen bzw. rechtlichen Elternteils mit dem Kind. Die Beziehung zwischen dem Patchwork-Elternteil und dem Kind wird deshalb vom Familienbegriff des Art. 6 Abs. I GG nicht erfasst. Denn neben der tatsächlichen Verantwortungsübernahme, die bei dem PatchworkElternteil vorliegt, ist für den verfassungsrechtlichen Schutz die rechtliche oder biologische Legitimation des Elternteils erforderlich. An einer solchen fehlt es aber beim Patchwork-Elternteil.

Hingegen ist die Patchwork-Familie im Familienrecht des BGB seit dem 30.04.2004, wenn auch nicht ausdrücklich, angekommen. Denn der PatchworkElternteil kann als enge Bezugsperson ein Umgangsrecht nach $\mathbb{\text { I } 6 8 5}$ A Abs. 2 BGB erlangen, falls die übrigen Voraussetzungen der Norm vorliegen. Das Familienrecht befindet sich hier weiter im Fluss. Der Gesetzgeber wird aufgrund der Veränderung der Familienstrukturen nicht umhin kommen, die Konstellation der Patchwork-Familie auch bei künftigen Gesetzesänderungen zu beachten.

Angesichts der veränderten Familienverhältnisse in der Gesellschaft darf diese Familienform auch im Haftungsalltag nicht übersehen werden. Im Rahmen der in $\ 832$ BGB speziell geregelten Elternhaftung ist sie wie die anderen Familien-

79 BVerfGE I08, 82 ( I I 7 ff.) v. 09.04.2003.

80 Gesetz zur Änderung der Vorschriften über die Anfechtung der Vaterschaft und das Umgangsrecht von Bezugspersonen des Kindes, zur Registrierung von Vorsorgeverfügungen und zur Einführung von Vordrucken für die Vergütung von Berufsbetreuern v. 23.04.2004, BGBl. I 2004, S. 598.

8I BT-Drucks. I $5 / 2253$, S. I I.

82 Luthin, FamRZ $2005,706$.

83 Schwab, Familienrecht, I3. A. (2005), Rn. 693. Der BGH hat nach In-Kraft-Treten der Vorschrift klargestellt, dass das Umgangsrecht nach $\ \mathrm{I}_{68} 5$ Abs. 2 BGB keinen aktuell bestehenden persönlich-vertrauten Bezug des Kindes zu der Bezugsperson zur Voraussetzung hat (FamRZ 2005, 705 f. v. 09.1 2.2005). 
formen zu behandeln. ${ }^{84}$ Aufgrund des gewandelten Familienbildes in der Gesellschaft ist $\$ 832$ Abs. 2 BGB analog auf den Patchwork-Elternteil für das Kind seines Partners anzuwenden. Er besitzt in der häuslichen Gemeinschaft für das Kind eine weitreichende Obhut von längerer Dauer mit weitgehender Einwirkungsmöglichkeit. Der Patchwork-Elternteil haftet daher - wie der sorgeberechtigte Elternteil - aus vermuteter Aufsichtspflichtverletzung, wenn das Kind seines Partners einen Schaden anrichtet. ${ }^{85}$

\section{Ergebnis}

I. Das Familienbild in Deutschland hat sich in den letzten Jahrzehnten grundlegend verändert (I.). Dieser Wandel hat sich auch im verfassungsrechtlichen Familienschutz (III. I.) und im Familienrecht des BGB (III. 2.) niedergeschlagen. Eine typische, gesellschaftlich akzeptierte Erscheinungsform des heutigen sozialen Lebens ist das nichteheliche Zusammenleben eines Elternteils mit einem Partner, der nicht der andere leibliche Elternteil des Kindes ist. Weder das Familienrecht noch das Deliktsrecht dürfen sich vor diesen gewandelten Familienverhältnissen und Gesellschaftsanschauungen verschließen (vor I.).

2. Es gibt bisher keine juristische Definition der Patchwork-Familie. Auch fehlt es an feststehenden Merkmalen, was eine solche kennzeichnet. Umgangssprachlich wird sie als Familie definiert, in der außer den gemeinsamen Kindern auch Kinder aus früheren Beziehungen der Mutter bzw. des Vaters oder beider Eltern leben. Dabei werden unter dem Begriff Patchwork-Familie sowohl Beziehungen erfasst, in denen die neuen Partner die Ehe geschlossen bzw. eine Lebenspartnerschaft begründet haben, als auch solche, in denen es sich um eine nichteheliche Lebensgemeinschaft handelt. Nach hier vertretener Auffassung ist die Patchwork-Familie im juristischen Sinn enger zu definieren, was sich durch ihre Abgrenzung von der Ehe, der Stieffamilie und der nichtehelichen Lebensgemeinschaft ergibt (II. I., 2.). Die Drei-Generationenfamilie fällt nicht unter den juristischen Begriff der Patchwork-Familie (II. 3). Hinsichtlich der Beziehungsintensität ist die Patchwork-Familie von der bloßen Wohn- und Hausgemeinschaft abzugrenzen (II. 3.).

3. Die Patchwork-Familie im juristischen Sinn ist eine im gemeinsamen Haushalt lebende nichteheliche Lebensgemeinschaft mit mindestens einem Kind, welches nur vom dafür sorgeberechtigten Partner abstammt. Die kleinstmögliche Patchwork-Familie setzt somit drei Personen voraus. Der nicht leibliche, sorgerechtslose Elternteil ist in Bezug auf das Kind des Partners als Patchwork-Elternteil zu bezeichnen. Bringt er selbst ein leibliches Kind mit in die Beziehung ein, wird diesbezüglich vom leiblichen Elternteil gesprochen. Heiraten die Partner, so handelt es sich um eine Stieffamilie (II. 4.).

4. Die Patchwork-Familie unterfällt in ihrer Gesamtheit nicht dem Familienbegriff des Art. 6 Abs. I GG. Den verfassungsrechtlichen Schutz genießt nur die Beziehung zwischen dem leiblichen bzw. rechtlichen Elternteil und dem Kind. Im bürgerlichen Recht ist die Patchwork-Familie jedoch bereits angekommen. So wird im Familienrecht der Patchwork-Elternteil vom Kreis der umgangsberechtigten Bezugspersonen des $\$ I685 Abs. 2 BGB erfasst. Im Deliktsrecht haftet er aus vermuteter Aufsichtspflichtverletzung gemäß $\$ 832$ Abs. 2 BGB analog für die vom Kind seines Partners verursachten Schäden (III. 3.). 